The conclusion that the gelatin functions differently in the two cases does not appear to be justified. T. R. Bolam.

Chemistry Department, The University, King's Buildings, Edinburgh, May 5.

2 NATURE, April 25, 1931, 127, 628.

2 Trans. Far. Soc., 22, $159 ; 1926$.

Trans. Far. Soc., 22, 166, 167; 1926.

- Trans. Far. Soc., 19, 285 ; 1923.

Wiтh reference to the letter by Mr. G. M. Nabar and Dr. B. N. Desai in NAture of April 25, p. 628, I should like to direct attention to the theory of banded precipitates that I published in various journals as the result of a series of experiments from 1916 to 1922, and summarised in a paper on "Adsorptive Stratification in Gels, V ". 1 In these papers the rôle of the ionic product and the metastable limit is fully discussed.

The authors also appear to be under the impression that silver chloride does not form Liesegang rings in gelatin; but this is not quite correct. The phenomenon is the same whatever the medium in which the precipitate is formed ; and as I have stated, "No serious attempt to obtain a sufficiently insoluble precipitate in the stratified form has failed. . . . There appears to be no evidence to show that stratification will not occur, provided a precipitate can be obtained in a suitable state of subdivision from solutions of sufficient dilution." Actually the formation of bands of silver chloride in dilute solution in gelatin is recorded by W. M. Fischer, ${ }^{2}$ bands of this substance in water in capillary tubes were obtained by Brodersen, ${ }^{3}$ and I obtained good bands in U-tubes in agar agar.4 The formation of banded precipitates of silver chloride, as with any other insoluble substance, for example, silver chromate in agar agar, depends primarily on adjusting the supersaturation produced so that a suitably finely divided precipitate will be obtained.

Science Museum, South Kensington, London, S.W.7.

I J. Soc. Chem. Ind., 7, 78; 1929 .

2 . Anorg. u. Allg. Chem., 145, 311 ; 1925.

Kolloid Z., 35, 21 ; 1924.

- Biochem. J., 10, 170; 1916.

By the courtesy of the Editor of Nature, I am able to give the following reply to the comments of Drs. Bolam and Bradford on our letter on " Condition of Silver Chromate in Gelatine Hydrolysed and Electro-Dialysed to Different Extents", published in NATURE of April 25, 1931, p. 628.

Drs. Bolam and Mackenzie ${ }^{1}$ have not given any experiments in which silver chloride rings in gelatine were obtained in the absence of silver chromate. In our experiments, the contact potential of the silver electrode reached its final value as soon as the solu. tions of silver nitrate in gelatine and potassium chloride in gelatine were mixed, a result which does not agree with the observations of Langdon. ${ }^{2}$ Using the same sample of gelatine, it was, however, found that with silver chromate the fall in the contact potential of the silver electrode was gradual. The contact potential measurements show that, in our experiments, silver chloride in gelatine was not in ionic condition, but that silver chromate was. $\mathrm{We}$ hold that, in the first case, gelatine acts as a protective colloid, and in the second case, as an inhibiting agent; and that it is the inhibitive power of gelatine which is responsible for the formation of Liesegang rings.

We agree with Dr. Bradford that serious attempts to obtain a sufficiently insoluble precipitate in the stratified form will prove successful. We also believe that just as bands of silver chloride in gelatine can be obtained by taking the former substance in sufficient dilution, the bands of the same substance in gelatine can be obtained even in slightly stronger solutions, provided the $p \mathrm{H}$ of gelatine is decreased sufficiently. We have found experimentally that the inhibitive power of gelatine towards silver chromate can be altered considerably even by slight changes in its $p \mathrm{H}$ value. We are at present studying the effect of changing $p \mathrm{H}$ of gelatine on its efficiency in giving banded precipitates (rings) of different sparingly soluble substances.

All the points raised in the communications of Drs. Bolam and Bradford are dealt with in detail in a paper which will be sent shortly for publication.

Physical Chemistry Laboratory,

Wilson College, Bombay, 7, July 3.

1 Trans. Far. Soc., 22, 159 ; 1926.

2 Trans. Far. Soc., 19, 285 ; 1923

\section{Oxidation Reduction Potential of Complex Iron Compounds in Yeast.}

WHEN an alkaline extract of yeast is saturated with ammonium sulphate there is precipitated, with the protein, cytochrome ' $\mathrm{C}$ ' and a complex iron compound giving no visible spectrum. The latter can be separated from the proteins slowly by ultra-filtration. It remains in solution when the protein, with the cytochrome ' $\mathrm{C}$ ', is precipitated by trichloracetic acid.

This iron compound is of interest because its oxidation potential $\left(E_{0}\right.$, referred to the normal hydrogen electrode, $=+0.02$ at $p H 7 \cdot 1)$ lies very close to that at which the spectrum of cytochrome ' $C$ ' appears and disappears - a potential near which a variety of aerobic organisms appear to function. In quite low concentration it prevents the hydrogen electrode from giving correct values in buffered solutions-a property which makes it seem likely that it can act as a catalyst.

That the iron is complexly bound and not in the form of simple ferrous or ferric ions is shown by the fact that even in a large excess of such a buffer as a concentrated borate tartrate mixture, the oxidation reduction potential of this compound lies about 0.25 volt above that of ferric chloride added to the buffer. The iron of the compound does not react with sulphocyanide when oxidised, but does react slowly with ad dipyrridyl when reduced.

An attempt to isolate the compound is now in progress. Because its oxidation potential is close to that of cytochrome ' $\mathrm{C}$ ', and because it is apt to accompany the latter in attempts to obtain the pure pigment, there is some danger of confusing the effects and properties of the two substances.

Converse Chemical Laboratory,

Thomas B. CoOLIDge.

\section{Cambridge, Mass., U.S.A., July 7.}

\section{Measurement of Ultra-Violet Radiation.}

WE are interested in the photographic method of recording the ultra-violet light of the sky, which has been described by Dr. J. R. Ashworth. ${ }^{1}$

With reference to the criticisms of Dr. Cunliffe ${ }^{2}$ the objection to the step-wedge does not appear to be important; it is probable that the logarithmic 'law' is approximately correct and in any case the wedge can be calibrated. It is, of course, well known that the photographic effect of an exposure is not a simple function of the time integral of the intensity 